\title{
VANISHING BONE DISEASE OF MANDIBLE: A CASE REPORT
}

\section{Joseph Edward ${ }^{1}$, Jacob John², Mubarak Aziz ${ }^{3}$, Veena Viswarajan ${ }^{4}$, Sanjith P Salim ${ }^{5}$}

\section{HOW TO CITE THIS ARTICLE:}

Joseph Edward, Jacob John, Mubarak Aziz, Veena Viswarajan, Sanjith P Salim. "Vanishing Bone Disease of Mandible: A Case Report". Journal of Evolution of Medical and Dental Sciences 2014; Vol. 3, Issue 23, June 09; Page: 6369-6377, DOI: $10.14260 /$ jemds/2014/2749

ABSTRACT: Vanishing bone disease is an extremely rare disorder of unknown aetiology. Vanishing bone disease presents as a progressive idiopathic osteolysis of one bone or contiguous bones around one focus, without respect for joint boundaries. More than 200 cases have been described in the international literature, with fewer than 42 involving maxillofacial site, usually the mandible. Disease of the jaws may manifest as pain, deformity, tooth mobility and malocclusion. We hereby report a case of 62 year old male patient with similar symptoms.

KEYWORDS: Gorham's disease; Massive Osteolysis; Disappearing Bone Disease; Phantom Bone Disease; Vanishing Bone Disease; Mandible.

INTRODUCTION: Massive osteolysis is a rare, insidious, chronic disease characterized by progressive resorption of contiguous osseous structures. First described in 1838 by Jackson, ${ }^{[1,2]}$ who reported the case of a young man with a progressively disappearing humerus, the syndrome became known as Gorham-Stout disease in 1955 when Gorham and Stout published a review of 24 cases. Facial involvement was first reported by Romer in 1924[3] and complete mandible lysis was reported by Thompson in 1933.The disease has many names: Gorham's disease; massive, spontaneous or progressive osteolysis; acute essential bone resorption and disappearing, phantom, or vanishing bone disease.

In most patients, the disease develops in the fourth decade of life, although it has been described in patients ranging in age from 18months to 72years. There is no genetic basis for the transmission of the disease, no sex or racial predilection and no associated endocrine disorders, but there is a predilection for the pelvis, humeral head, humeral shaft, axial skeleton, and mandible. To date more than 200 cases have been described in the international literature, with fewer than 42 involving a maxillofacial site, usually the mandible.

The indicated disease may or may not be painful and is most commonly detected after fractures or history of injury. When the jaw, tooth sockets, neck, face and head are affected, possible symptoms include loosening of teeth, fractures, and pain. Because early diagnosis is crucial to prevent significant morbidity and in some cases even mortality, surgeons should consider including Gorham's disease among the differential diagnosis of pathological entities leading to tooth mobility and later osteolysis.

CASE REPORT: A 62 year old male patient reported to our dental OPD complaining of pain and swelling of 2 week's duration over the left side of the face which was treated elsewhere. History of traumatic extraction of lower left molars underwent 3 months before present complaint. On examination, a $10 \times 8 \times 5 \mathrm{cms}$ firm to hard tender swelling anteroposteriorly extending from anterior border of masseter to the posterior border of mandible and superoinferiorly extending from 
zygomatic arch to lower border of mandible with no inflammatory changes and mild lymphadenopathy (Fig. 1).

On intra oral examination, bilateral leukoplakia like patches on buccal mucosa and retro molar areas were identified. Limited mouth opening of $21 \mathrm{~mm}$ was present. Multiple missing teeth following extraction of,4,5,6,7,8,9 was identified. Repeated incisional biopsy for leukoplakia patches from different centers gave report of hyperplastic epithelium.

OPG then took, revealed an extensive ill -defined radiolucency of body of mandible extending from 37 regions involving angle and extending up the ascending ramus of mandible (Fig. 2). On haematological test, only erythrocyte sedimentation rate was raised ( $45 \mathrm{~mm} /$ hour). To rule out an Intraosseus lesion, bone biopsy was performed which claimed to be as Giant cell lesion.

CT scan took, showed an ill- defined radiolucent lesion extending from the body of mandible to ascending ramus of mandible (Fig. 3). Differential diagnosis included infectious diseases like osteomyelitis, central giant cell granuloma; inflammatory conditions like Rheumatoid arthritis, secondaries of jaws, osteogenic sarcoma; Histocytosis, Stills disease, endocrine disorders like hyperparathyroidism that needed to be excluded.

There was no anemia or leucocytosis, but Liver function test, serum urea and creatinine were within normal limits. Serum alkaline phosphatase and parathyroid hormone levels were within normal limits. Mycological and bacteriological evaluation was negative. Chest radiograph was normal. As the bone biopsy findings were inconclusive, surgical exploration biopsy via submandibular approach was planned and done (Fig. 4). Intraoperatively, extensive osteolysis of mandible from premolar region to mid ramal region was noted (Fig. 5, 6).

A cloudy dark viscous fluid drained out from the site was sent for culture and found to be sterile. Thorough wound debridement and curettage was done. Histopathological examination of the lesion showed fibrous connective tissue with many thin walled vascular spaces and minimal chronic inflammatory infiltrate with no features of cellular atypia and was suggestive of an angiomatous lesion (Fig. 7).

Patient was lost to follow up for 3months again, following which he reported back with the recurrence of swelling in left angle of mandible with trismus and pain and an OPG was taken. On the comparison with earlier radiological investigations osteolysis had extended further to involve condyle (Fig. 8). Hematological picture was normal again. Systemic evaluation was done to rule out other pathological causes of osteolysis.

The positive biopsy result combined with osteolytic radiographic pattern; clinical signs; lack of involvement of other organs and the absence of etiological factors (neoplastic; immunological; metabolic; hereditary or infectious) helped confirm the diagnosis of Gorham Stout disease. Patient was managed with surgical resection of the lesion with a clear margin from body of mandible to condyle and disarticulation of the left side of mandible with Submandibular and Hinds approach. Surgical resection was followed by local drug delivery with Metronidazole for 5days (Fig. 9, 10, 11) and administration of parenteral IV antibiotics and analgesics was given. Histopathological examination of the resected mandible revealed an angiomatous lesion similar to that of previous biopsy report with no cellular atypia (Fig. 12).

Postoperatively, patient was asymptomatic. On follow up, mouth opening of the patient was improved to $31 \mathrm{mms}$ (Fig. 13). Post-operative OPG taken after 3months showed no evidence of further osteolysis (Fig. 14). Now the patient is under regular follow up every 6 months. 
DISCUSSION: The term used by Gorham and Stout1 was "haemangiomatosis, " implying a proliferative process. According to Gorham and Stout, the most characteristic histologic abnormality in massive osteolysis is the change of bone into connective tissue. This connective tissue contains many thinwalled vessels, sometimes with red blood cells. In other areas, freely anastomosing vascular spaces lined by endothelial cells can be seen.

The fatty marrow also contains some dilated blood vessels. Johnson and McClure in 1958 reported on the relative frequency of massive osteolysis in different bones. The sequence is as follows: clavicle, scapula, proximal end of humerus, ribs, iliac bone, ischium and sacrum. The disease has not been observed in the calvaria or in the distal bones of the extremities.

Based on the radiological, morphological and clinical findings in previous reports, we diagnosed this case as an idiopathic osteolysis, distinguishable from the many local and systemic conditions which are associated with bone resorption (secondary osteolysis). These include disuse atrophy, acute inflammatory atrophy associated with trauma (Sudeck's atrophy or algodystrophy), primary and metastatic tumours, hyperparathyroidism, gout, congenital pseudarthrosis, granulomatous diseases, rheumatoid arthritis, diabetes mellitus, psoriatic arthritis, osteomyelitis, systemic mastocytosis, aseptic necrosis, neurogenic arthropathy, prolonged steroid therapy, bony aneurysm, and cystic angiomatous of bone. Idiopathic osteolysis comprises a heterogeneous group of rare diseases, characterized by the spontaneous onset of mostly peripheral osteolysis, without obvious reason.

It has to be differentiated from familial and sporadic cases and uneccentric osteolysis. Hardegger, Simpson and Segmuller ${ }^{[10]}$ proposed a classification with five types of the idiopathic osteolysis, based on the reports of Torg et al.[11] and Macpherson, Walker and Kowall.[12] Multicentric osteolysis can be divided into a hereditary form with either dominant or recessive transmission and a non-hereditary variety with nephropathy.

It usually presents in childhood or adolescence. The Gorham-Stout syndrome and the Winchester syndrome belong to the monocentric types of osteolysis. The patient whom we presented had monocentric osteolytic changes as adult; there was no family history of bone disease or of renal involvement. According to the classification proposed by Hardegger et al they belong to a group defined as the Gorham-Stout syndrome.

The exact pathogenic mechanism of Gorham- Stout syndrome is still unknown. There is controversy even over the presence or absence of osteoclasts in the condition. Several authors believe that angiomatosis is responsible. Knoch[13] suggested that a previous silent hematoma becomes active after a minor trauma and leads to resorption of bone. Neurovascular changes, like those seen in Sudeck's atrophy, have been described. Thompson and Schurman[14] suggested that the disease is a primary aberration of vascular tissue in bone, related to hyperaemic granulation tissue. Young et al[15] believed that osteolysis is attributable to a basic underlying endothelial dysplasia of lymphatics, blood vessels or both. Heyden et al suggested that angiomatosis might induce local hypoxia and acidosis and that this might favor the activity of local hydrolytic enzymes.

The osteolytic process is often painless allowing patients to continue with daily activities while bone destruction progresses, making the patient more susceptible to pathological fractures.

Heffez et al[16] produced a set of diagnostic criteria to aid diagnosis:

1. A positive biopsy.

2. Absence of cellular atypia. 
3. Minimal/no osteoblastic response and the absence of dystrophic calcification.

4. Evidence of local, progressive osseous resorption.

5. Non-expansile, non-ulcerative lesion.

6. Absence of visceral involvement.

7. Osteolytic radiographic pattern.

8. Negative hereditary, metabolic, neoplastic, immunologic or infectious aetiology.

In a review by Anavi et al,[17]the mean duration of symptoms before diagnosis was 6.4 years with the initial histopathologic diagnosis inaccurate in $45 \%$ of cases.

Radiographic features according to Resnick et al[18] during the initial stage of the lesion, radiolucent foci appear in the intramedullary or subcortical regions, resembling findings seen in patchy osteoporosis. Subsequently, slowly progressive atrophy, dissolution, fracture, and disappearance of a portion with tapering or "pointing" of the remaining osseous tissue and atrophy of soft tissues. Our case was diagnosed based on Heffez et al criteria and radiograph pattern similar to as proposed by Resnick et al.

Currently, there is no recognized effective treatment for this disease. Surgical, radiation and medical therapies have been used with variable outcomes. Resection and bone grafting have always resulted in recurrences and failures. Radiation therapy can be effective but its complications should be very carefully considered, especially in the child. Definitive radiation therapy in moderate doses (40-45 Gy in 2-Gy fractions) appears to result in a good clinical outcome with few long-term complications.

The medical treatment for Gorham disease includes alfa-2b interferon. More recently, first generation bisphosphonates have been reported to successfully arrest the course of osteolysis. Zoledronic acid, a nitrogen-containing high-potency bisphosphonate, has been reported to be more potent and effective than the first generation bisphosphonates and to have anti-angiogenic properties in addition to osteoclast inhibition.

It has been proposed that this therapy could potentially be used in managing the active phase of the disease and could be offered as an alternative to radiotherapy. However, recently, it has been established that this mode of treatment is not without complications, as bisphosphonates administered intravenously and orally have both been associated with osteonecrosis of the jaws. In recent years, most patients have been treated with surgery and/or radiation therapy.

CONCLUSION: Gorham Stout disease is an intriguing condition. Several publications in the literature have reported difficulties in diagnosing this disease owing to its insidious onset and successive phases of bone destruction over a period of several months. Histological examination does not give an actual diagnosis but is the essential criteria for exclusion of other pathological conditions. Positive diagnosis of this rare entity is based on the exclusion of other pathological entities.

Various treatment modalities have been suggested of which surgical resection and local drug delivery system helped in our case to arrest the progression of the disease. Prognosis of the disease is unpredictable and needs long- term evaluation. 


\section{REFERENCES:}

1. Jackson JBS (1838) A boneless arm. Boston Med Surg J 18:368-369.

2. Jackson JBS (1872) Absorption of humerus after fracture. Boston Med Surg J 10:245-247.

3. Romer $O$ (1924) Die pathologic der Zahne, in von Henke F, Lubarsch $O$ (eds): Handbuch der 499.

4. Dunbar SF, Rosenberg A, Mankin H (1993) Gorham's massive osteolysis: The role of radiation therapy and a review of the literature. Int J Radiat Oncol Bio Phys 26: 491,

5. Vinee P, Tanyu MO, Hauenstein KH (1994) CT and MRI of Gorham syndrome. J Comput Assist Tomogr 18:985

6. Johnson PM, McClure JG (1958): Observations on massive osteolysis. A review of the literature and report of a case. Radiology 71: 28.

7. Gutierrez RM, Spjut HJ (1972) Skeletalangiomatosis: Report of three cases and review of the literature. Clin Orthop Relat Res 82.

8. Halliday DR, Dahlin DC, Pugh DG (1964) Massive osteolysis and angiomatosis. Radiology 82: 637.

9. Kery L, Wouters HW (1970) Massive osteolysis Report of two cases. J Bone Joint Surg Br. 52; 452. 10. Hardegger F, Simpson LA, Segmueller G (1985). The syndrome of idiopathic osteolysis: classification, review and case report. J Bone Joint Surg [Br]; 67-B: 89-93.

10. Torg JS, Steel HH (1969) Sequential roentgenographic changes occurring in massive osteolysis J Bone Joint Surg Am 51: 1649

11. Walker RD, Kowall MH (1973) Essential osteolysis with nephropathy. J Can Assoc Radio l; 24: 98-103.

12. Knoch H-G(1963)Die Gorhamsche Krankheitausklinischer Sicht. Zentralbl Chir; 18:674-83.

13. Thompson JS, Schurman DJ. (1933) Massive osteolysis: case report and review of the literature. Clin Orthop; 103: 206-11. Disappearing bone disease: A clinical and histological study. J Bone Joint Surg Am 59:57.

14. Young JW, Galbraith M, Cunningham J(1983). Progressive vertebral collapse in diffuse angiomatosis. Metab Bone Dis Relat Res; 5: 53-60.

15. Heffez L, Doku HC, Carter BL (1983): Perspectives on massive osteolysis. Report of a case and review of the literature. Oral Surg Oral Med Oral Pathol 55:331 Vélez A, Herrera M, Del Rio E(1993) Gorham's syndrome. Int J Dermatol 32:884.

16. Anavi Y, Sabes WR, Mintz S (1989) Gorham's disease affecting the maxillofacial skeleton. Head Neck 11:550.

17. Resnick D: Osteolysis and chondrolysis, in Resnick D (2002): Diagnosis of Bone and Joint Disorders (ed 4). Philadelphia, PA, Saunder, pp 4920-4944. 
Figure 1: Initial Presentation of the patient.

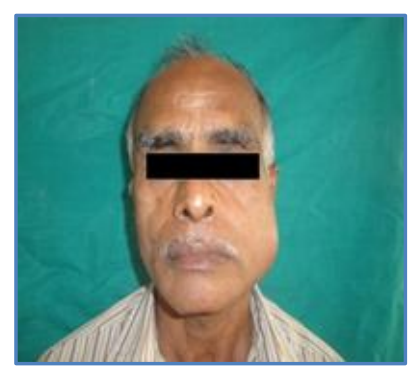

\section{Figure 1}

Figure 2: OPG taken revealed an extensive ill-defined osteolytic lesion of body of mandible extending from 37 regions involving angle and extending up the ascending ramus of mandible.

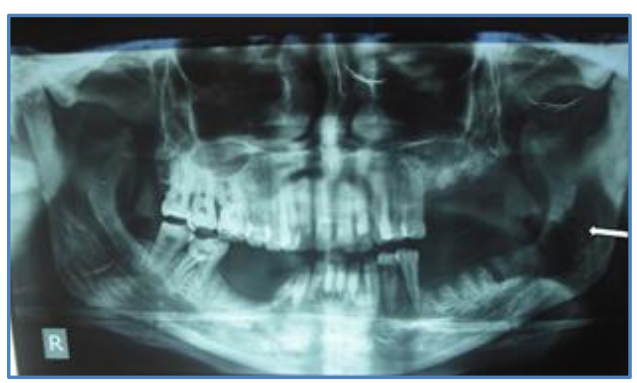

Figure 2

Figure 3: CT showing extent of osteolytic lesion.

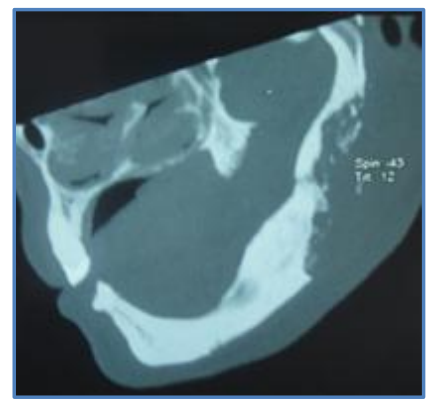

Figure 3

Figure 4: Shows extended Submandibular lesion incision

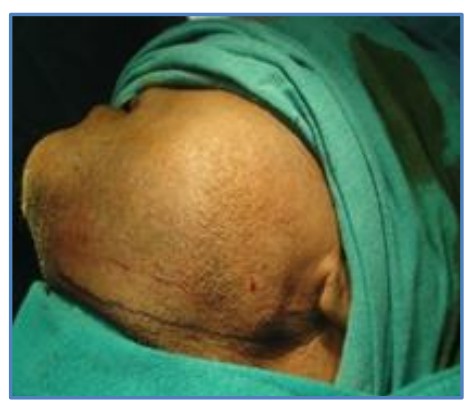

Figure 4 


\section{CASE REPORT}

Figure 5: Note the osteolytic fluid draining from the mandible

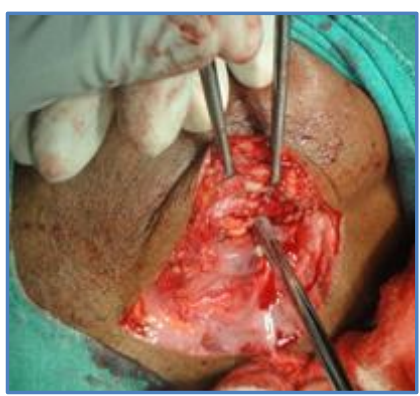

\section{Figure 5}

Figure 6: Shows missing part of mandible from the body to the condylar region

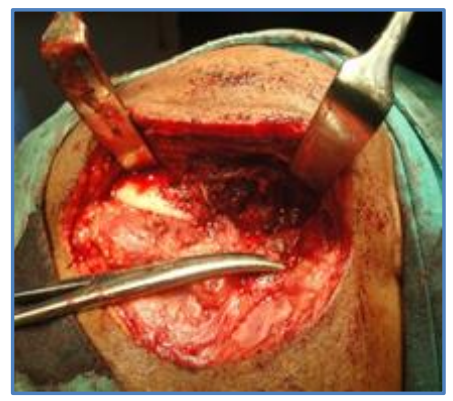

\section{Figure 6}

Figure 7: Section shows fibrous connective tissue with numerous thin walled vascular spaces, minimal chronic inflammatory infiltrate. No cellular atypia seen (H \& E 10X)

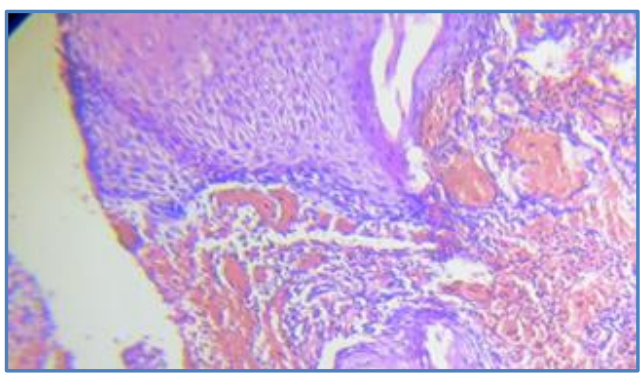

\section{Figure 7}

Figure 8: Shows the osteolyic lesion extending to left condylar region.

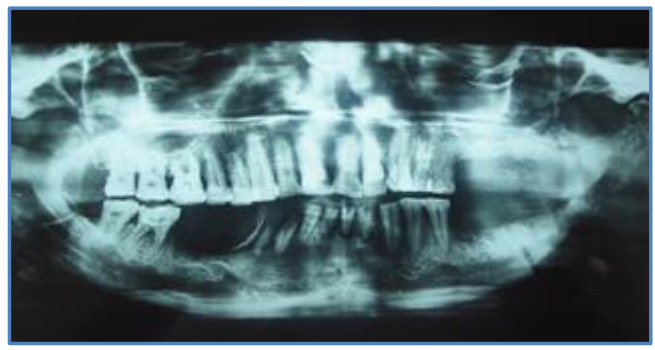

\section{Figure 8}




\section{CASE REPORT}

Figure 9: Shows combined Submandibular and Hinds approach.

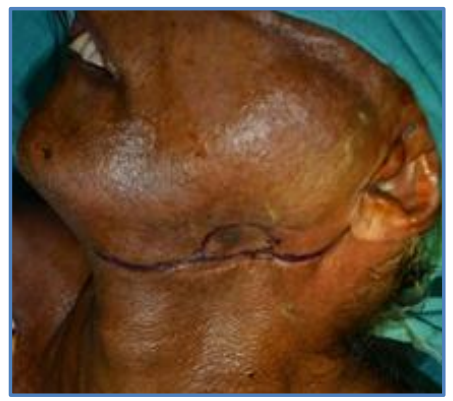

\section{Figure 9}

Figure 10: shows resected part of mandible after thorough curettage.

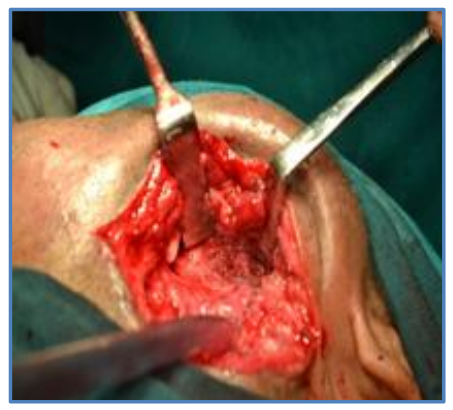

Figure 10

Figure 11: Metronidazole local drug delivery system in place.

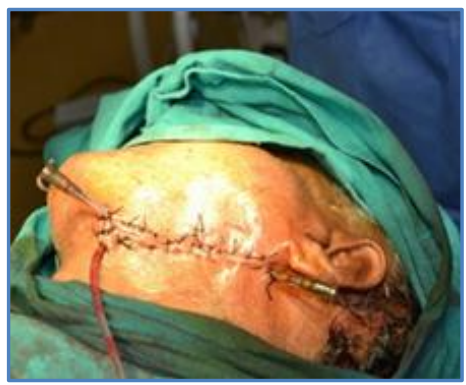

Figure 11

Figure 12: Histopathological examination of resected mandible which revealed an angiomatous lesion with no cellular atypia.

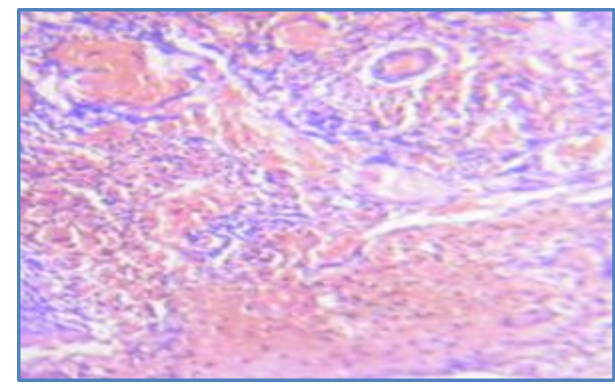

Figure 12 


\section{CASE REPORT}

Figure 13: Postoperative review with resolved trismus.

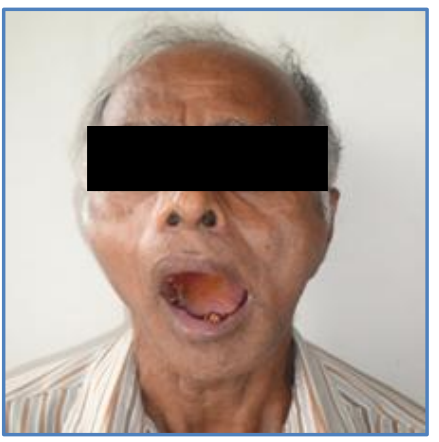

\section{Figure 13}

Figure 14: Post op OPG showing the resected areas of mandible.

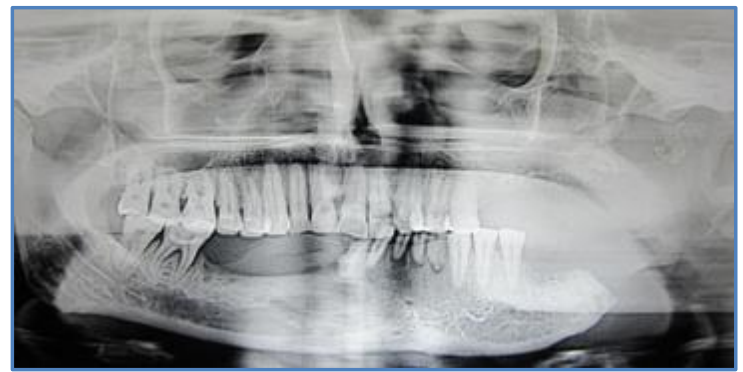

Figure 13

\section{AUTHORS:}

1. Joseph Edward

2. Jacob John

3. Mubarak Aziz

4. Veena Viswarajan

5. Sanjith P Salim

\section{PARTICULARS OF CONTRIBUTORS:}

1. Professor and HOD, Department of Oral and Maxillofacial Surgery, Azeezia College of Dental Sciences and Research.

2. Associate Professor, Department of Oral and Maxillofacial Surgery, Azeezia College of Dental Sciences and Research.

3. Senior Lecturer, Department of Oral and Maxillofacial Surgery, Azeezia College of Dental Sciences and Research.
4. Final Year PG Student, Department of Oral and Maxillofacial Surgery, Azeezia College of Dental Sciences and Research.

5. II ${ }^{\text {nd }}$ Year PG Student, Department of Oral and Maxillofacial Surgery, Azeezia College of Dental Sciences and Research.

\section{NAME ADDRESS EMAIL ID OF THE CORRESPONDING AUTHOR:}

Dr. Mubarak Aziz,

Department of OMFS,

Azeezia College of Dental Sciences and Research, E-mail: mubarakaziz@gmail.com

Date of Submission: 14/05/2014.

Date of Peer Review: 15/05/2014.

Date of Acceptance: 20/05/2014.

Date of Publishing: 05/06/2014. 